\title{
Effect of Nursing Intervention for Secondary School Students about Substance Abuse Prevention in Sammanoud City, Gharbia Governorate, Egypt
}

\author{
Dr. Aliaa M. El-Afandy and Dr. Eman M. Hassan \\ ${ }^{I}$ Aliaa M. El-Afandy lecturer in Community Health Nursing Department, Faculty of Nursing, Helwan University, \\ Egypt \\ ${ }^{2}$ Eman M. Hassan lecturer in Pediatric Nursing Department, Faculty of Nursing, Helwan University, Egypt
}

\begin{abstract}
:
Background: Alcohol and other forms of substance abuse continue to hinder the development, functioning, and well-being of millions of adolescents and adults.

Purpose of the study: The aim of this study is to evaluate the effect of nursing intervention for secondary school students about substance abuse prevention in Sammanoud City, Gharbia Governorate, Egypt.

Subjects and methods: Design: A quasi experimental research design was used.

Setting: The study was conducted at secondary governmental schools in Sammanoud City, Gharbia Governorate, Egypt.

Sampling: A multistage cluster random sample of student from first grade secondary school students

Tool: An interview questionnaire form, that cover four parts as 1) demographic characteristics. 2) Knowledge and awareness, and 3) Attitude toward substance abuse and abuser, and 4) prevalence and experience of students toward smoking and substance abuse

Results: Highly statistically significant improvement was found among secondary school students knowledge and attitude toward substance abuse and abuser after the nursing intervention implementation Conclusion: The nursing intervention helped in improving students' knowledge, awareness, and their attitude about substance abuse prevention.

Recommendation: The study can be replicated with a larger number of students for generalizations of the results, more education with students at an earlier age than traditionally provided. Simultaneously, parents should also receive information on substances used by young people, the associated dangers with use, and the parents role in prevention of problem substance use.
\end{abstract}

Keywords: Secondary school students, substance abuse, nursing intervention, Sammanoud City, Egypt.

\section{Introduction:}

Teenage is a stage when a human being develops physically and mentally with a great risk for beginning substance use. Reproductive and child health program reported that teenagers are between 10-19 years old with population of 1.2 billion adolescents in the world. Experiments with addictive substances begin on adolescents because they are still immature from a cognitive point of view and they can be easily exposed to social influences. Current reports approved a worldwide increase in the availability of drugs and consumption among youth who are between 15 and 25 years old with percent (12.5\%-84\%) 1 .

The abuse of drugs has globally increased and it begins in a progressive age. The common reasons for drug abuse among teenagers are the encouragement from the group of friends, the desire for popularity, the poor control from the side of parents and the easy availability of drugs. There are other causes which include curiosity, enjoying specific feelings such as energy and confidence, risk taking and the excitement of rebellion especially when it becomes related to parents' disapproval and escapism 2.

Students go to school to overcome the evil of ignorance and the teachers shouldn't feel ashamed if they can't fix homelessness or violence or prevent AIDs or end taking drugs. Teachers will care when students use drugs or have sexual diseases but it isn't the concern of schools. Schools aren't concerned to repair such bad behaviors but they can influence that behavior through developing knowledge, skills and values in students 3 .

Smoking tobacco is common worldwide. The World Health Organization has approved that tobacco and its products kill more than 3.5 million people every year and it's predicted that by the decade 2020-2030, tobacco will kill 10 million people every year 4 .

Tobacco, alcohol and other legal or illegal kinds of drugs should be explained in educational programs for preventing them and should consider drug using levels among individuals and in society, risks and factors of protection, gender, culture, language, religion and sexual orientation. Teachers in schools should deal with students in a way that make them involved into knowledge of drug abuse prevention programs. Students react in 
appositive way when their needs and the users and non-users needs are acknowledged and communication channels are kept open 5.

Nurses have an important role in screening, brief intervention and referral for treatment for substance use disorders, through developing educational intervention program, they Provide information regarding effects of addiction on mood and personality, be alert to changes in behavior, provide positive feedback for expressing awareness of dangers of substance abuse, encourage and support patient's taking responsibility for own recovery, assist patient to learn own responsibility for recovering, encourage family members to seek help whether or not the abuser seeks it, and review program rules, philosophy expectations 6.

\section{Significance of the study:}

The early beginning of using drugs is found to have impairment, crime, and difficulty in giving up and has a relation with poor prediction and pattern of deceit and irresponsibility, people who drink are victims of violent crimes and they are exposed to be assault sexually and unwanted pregnancy. They also can face strong problems in school or can be involved in traffic crashes and have later problems because of drinking alcohol Drugs abuse is common among the teenagers worldwide 7.

\section{Aim of The Study}

The current study aimed to evaluate the effect of nursing intervention for secondary school students about substance abuse prevention in Sammanoud City, Gharbia Governorate, Egypt. Through the following objectives

1. Studying the use of some substances among the study group; assessing knowledge about drugs; and evaluating attitude toward substance abuse and abusers.

2. Planning, implementing and evaluating a nursing intervention of addiction prevention to improve knowledge and attitude of the studied sample.

Hypothesis: After implementation of the nursing intervention improvement in students' knowledge and their attitude about substance abuse prevention can be achieved.

Design:

\section{Subjects And Methods}

A quasi experimental design was used to conduct this study.

Setting:

The study was conducted at governmental secondary schools in Sammanoud City, Gharbia Governorate, Egypt, where Sammanoud City contain 13 general secondary schools with 2257 students in first grade, one technical secondary school with 1280 students in first grade, one agricultural secondary school with 620 students in the first grade, and seven commercial secondary schools with 555 students in the first grade in a total of 22 schools and 4712 students.

Sampling: A multistage cluster random sampling was used to select a sample of 385 school students having the following inclusion criteria: registered in the first grade, and willing to participate in the study.

\section{Sample Size Calculation:-}

The sample size was calculated using the following equation: [36]8.

At $95 \%$ confidence power of the study.

Steven Thimpsone equation $\mathrm{n}=$

Where:

$\mathrm{N} \times \mathrm{P}(1-\mathrm{P})$

$\mathrm{n}=$ Sample size

$\mathrm{N}=$ Total society size

$\mathrm{d}=$ Error percentage $=(0.05)$

$\mathrm{P}=$ Percentage of availability of the character and objectivity $=(0.5)$

$\mathrm{Z}=$ The corresponding standard class of significance $95 \%=(0.5)$

The calculated sample was 350 secondary school students at first grade. They were chosen randomly as a probability sample, out of total 4712 secondary school students. 35 students were included in the pilot study and they were excluded from the total sample of the study. So, the sample size included 350 secondary school students as a stratified random sample continuo to participate in the study.

\section{Tools for data collection:}

Data collection was carried out by using the following tool:

An interview questionnaire form that covers four parts: 
Part 1: Demographic characteristics such as: age, father and mother education.

Part 2: Designed by the researcher, to assess students' knowledge as: definition of substance abuse, causes, etc.

\section{Scoring system:}

Each knowledge question answers were categorized into don't know (scored zero), incomplete answer (scored one), and complete answer (scored two), the total knowledge scores ranged from 0-12, Results were evaluated as follows:

- Poor knowledge $(<50 \%)$ (with scores ranged from 0-6)

- Fair knowledge (50-75\%) (with scores ranged from 7-9)

- Good knowledge (>75\%) (with scores ranged from 10-12)

Part 3a: Designed by Al-Dossary( 2004) 9, it was used to assess student attitude regarding substance abuse using Likert scale

\section{Scoring system:}

- Pretest questions from 1-13 were scored from (0-2) (0 for don't agree, 1 for in- between, and 2 for agree).

- The total attitude scores ranged from (0-26). Results were evaluated as follows:

- Negative attitude $(<40 \%)$ (with scores ranged from $0-10)$

- Neutral attitude (40-60\%) (with scores ranged from 11-16)

- Positive attitude (>60\%) (with scores ranged from 17-26)

Part 3b: Designed by Al-Dossary (2004) 9, it was used to assess student attitude regarding substance abuser using Likert scale

\section{Scoring system:}

- Pretest questions from 1-18 were scored from (0-2) (0 for don't agree, 1 for in- between, and 2 for agree).

- The total attitude scores ranged from (0-36).

Results were evaluated as follows:

- Negative attitude (<40\%) (with scores ranged from 0-14)

- Neutral attitude (40-60\%) (with scores ranged from 15-22)

- Positive attitude (>60\%) (with scores ranged from 23-36)

Part 4: It was used to assess prevalence and experience of the studied secondary school about smoking and drug abuse.

\section{Pilot study}

A pilot study was carried out on $10 \%$ of the sample from different schools, to evaluate the clarity and applicability of the tool and necessary modifications were done based on their responses. The pilot study was also used to estimate the time needed to fill in the tool. Students who participated in the pilot study were later excluded from the main study sample.

\section{Validity:}

The study tool was tested for its content and face validity by a jury of five experts in the field of Community and Pediatric Health Nursing to evaluate items as well as the entire instrument as being relevant and appropriate in terms of the construct and if the items adequately measure all dimensions of the construct. The face validity of the questionnaire was calculated based on experts' opinion after calculating content validity index $(\%)$ of its items and it was $96 \%$.

\section{Reliability:}

The study tool was subjected to assessment of internal consistency reliability using Spearman-Brown Prophecy Formula $\left(r^{1}=2(3) / 1+r\right)$, where $r^{1}$ estimated reliability of the entire test and $r$ estimated correlation coefficient computed on the split halves. The study tool was tested by the pilot subjects at first session and retested after 2 weeks as test-retest reliability for calculating Cronbach's Alpha which was 0.877.

\section{Statistical analysis:}

The collected data were organized, tabulated and statistically analyzed using SPSS software Statistical Package for the Social Sciences, (SPSS) version 16, (Chicago, IL, USA). For quantitative data, the range, mean and standard deviation were calculated. For qualitative data, comparison between two groups and more was done using Chi-square test $\left(^{2}\right.$ ). For comparison between means of two groups of parametric data of independent 
samples, student t-test was used. For comparison between means of two related groups (pre and post program data) of parametric data, paired t-test was used. For comparison between more than two means of parametric data, F value of ANOVA test was calculated. Correlation between variables was evaluated using Pearson's correlation coefficient (r). Significance was adopted at $\mathrm{p}<0.05$ for interpretation of results of tests (Dawson \& Trapp, 2001).

\section{Field work:}

An official letter issued from the Faculty of Nursing, Helwan University was sent to the Ministry of Education and after that the executive of the schools, including the point and timetable of the review to get consents to visit schools and direct the review. The health education intervention about addiction prevention was created in view of audit of related writing and apparatus appraisal (pre-test). Time plan was set up and the understudies were sorted out into 6 bunches (20-30 students).

The program was executed amid school day began from 8.00 a.m. to 2.00 p.m. and instructive program built included 4 stages:

1) Preparatory stage: The preliminary stage was done by utilizing the assessment tools after being revised and tested for general information about substance abuse prevention. Time expended for answering the study sheet ranged from 30-45 minutes. This phase lasted for 2 weeks.

2) Planning stage: Based on the outcome acquired from the assessment Phase. The educational sessions were created after reviewing of related literature. Detected needs, requirements and deficiencies were converted into aim and objectives of the educational sessions and an illustrated booklet was created by the researchers and offered to every member as reference.

3) Program implementation stage: The booklet was implemented in the form of sessions. The length of every session was distinctive according to students' response, time accessible, and the content of each session.

4) Evaluation phase: Evaluation of the educational sessions was done quickly after its Implementation by contrasting the change in students' level of knowledge, and attitudes through applying the similar apparatuses of pre-test as posttest.

Ethical considerations: Consent to participate in the study was obtained from the parents of school students of the study group. They were given an opportunity to refuse to participate and they were notified that they can withdraw at any stage of research without giving any reason. Also they were assured that, the information given will remain confidential and used for the research purpose only.

\section{Result}

Table (1): Demographic characteristics of the studied Secondary school students $(n=350)$.

\begin{tabular}{|c|c|c|}
\hline \multicolumn{2}{|c|}{ Studied Secondary School Students $(n=350)$} & \multirow[t]{2}{*}{ Variables } \\
\hline$\%$ & No & \\
\hline & & Age in ( years): \\
\hline \multirow{2}{*}{\multicolumn{2}{|c|}{$\begin{array}{l}16-17 \\
16.20 \pm 0.40\end{array}$}} & Range \\
\hline & & Mean \pm SD \\
\hline & & School type: \\
\hline 56.3 & 197 & General secondary school \\
\hline \multirow[t]{2}{*}{43.7} & 153 & Technical school \\
\hline & & Fathers' education level: \\
\hline 2.0 & 7 & Illiterate \\
\hline 13.1 & 46 & Read \& write \\
\hline 8.3 & 29 & Basic education \\
\hline 37.7 & 132 & Middle education \\
\hline \multirow[t]{2}{*}{38.9} & 136 & University education \\
\hline & & Mothers' education level: \\
\hline 0.6 & 2 & Illiterate \\
\hline 9.1 & 32 & Read \& write \\
\hline 9.7 & 34 & Basic education \\
\hline 46.9 & 164 & Middle education \\
\hline \multirow[t]{2}{*}{33.7} & 118 & University education \\
\hline & & Residence: \\
\hline 39.4 & 138 & Rural \\
\hline 60.6 & 212 & Urban \\
\hline
\end{tabular}

Demographic characteristics of the secondary school students represented that, more than half $(56.3 \%)$ were in general secondary school. Regarding level of father education, 38.9\% of them had university education, while $46.9 \%$ of mothers had middle educational level. As regards residence, $60.6 \%$ of them were from urban. 


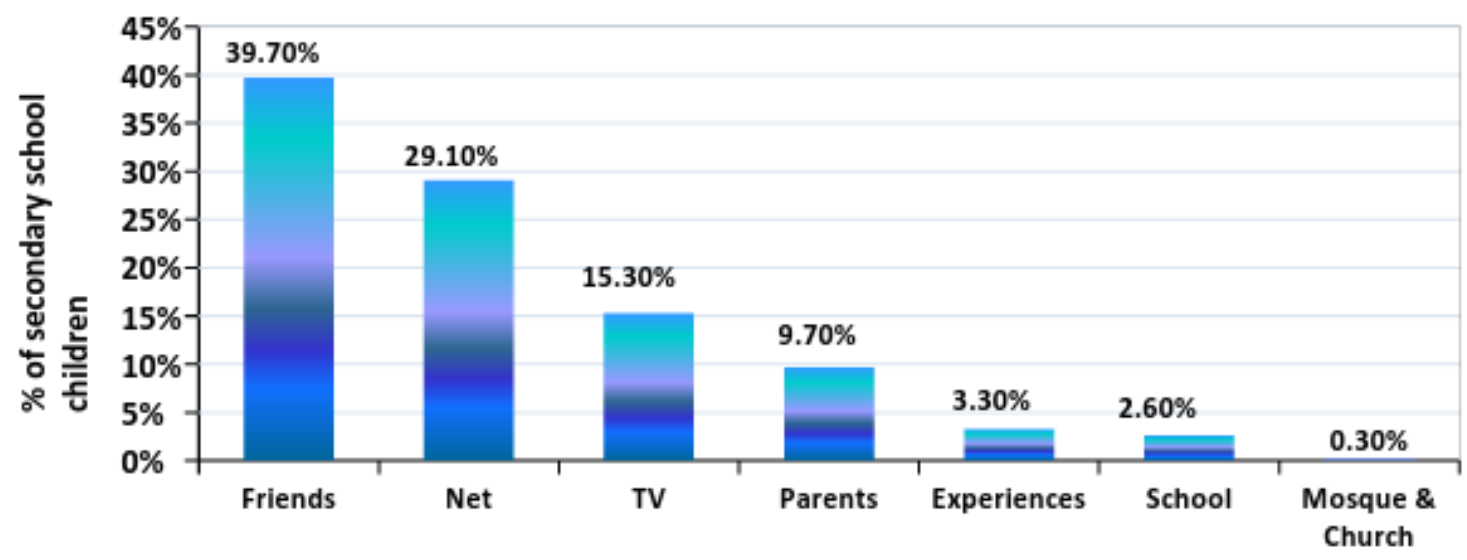

Figure (1): Sources of information of the studied secondary school students about substance abuse ( $\mathrm{n}=350)$.

Figure (1) represents that $39.7 \%, 29.1 \%$ and $15.3 \%$ estimated that their sources of information were acquired from friends, net, and T.V respectively, while least sources were acquired from parents, schools, experiences, mosques and churches.

Table (2): Mean scores and levels of total knowledge of the studied secondary school students about substance abuse pre/post intervention $(\mathrm{n}=350)$.

\begin{tabular}{|c|c|c|c|c|c|c|c|}
\hline \multirow[t]{3}{*}{$\begin{array}{l}\chi^{2} \\
\mathbf{P}\end{array}$} & \multicolumn{4}{|c|}{$\begin{array}{l}\text { The Studied Secondary School Students } \\
(n=350)\end{array}$} & \multicolumn{3}{|c|}{$\begin{array}{l}\text { Total Knowledge About } \\
\text { Substance Abuse }\end{array}$} \\
\hline & \multicolumn{2}{|c|}{ Post- intervention } & \multicolumn{2}{|c|}{ Pre-intervention } & & & \\
\hline & $\%$ & No & $\%$ & No & & & \\
\hline & & & & & \multicolumn{3}{|c|}{ Levels of total knowledge: } \\
\hline 605.011 & 0.0 & 0.0 & 86.6 & 303 & $(0-6)$ & $(<50 \%)$ & Poor \\
\hline \multirow[t]{5}{*}{$0.0001^{*}$} & 13.7 & 48 & 13.4 & 47 & $(7-9)$ & $(50-<75 \%)$ & Fair \\
\hline & 86.3 & 302 & 0.0 & 0.0 & $(10-12)$ & $(\geq 75 \%)$ & Good \\
\hline & & & & & \multicolumn{3}{|c|}{ Total knowledge scores: } \\
\hline & \multicolumn{2}{|c|}{$\begin{array}{l}8-12 \\
11.50 \pm 1.13\end{array}$} & \multicolumn{2}{|c|}{$\begin{array}{l}0-9 \\
4.48 \pm 2.19\end{array}$} & \multicolumn{3}{|c|}{$\begin{array}{l}\text { Range } \\
\text { Mean } \pm \text { SD }\end{array}$} \\
\hline & \multicolumn{4}{|c|}{$\begin{array}{l}80.056 \\
0.0001 *\end{array}$} & \multicolumn{3}{|c|}{$\begin{array}{l}\text { Paired t-test } \\
\mathrm{P}\end{array}$} \\
\hline
\end{tabular}

\section{*Significant $(\mathbf{P}<0.05)$}

Highly statistically significant improvement was found among secondary school students knowledge after the nursing intervention implementation $(\mathrm{P}=0.0001)$.

Table (3): Knowledge items of the studied secondary school students about substance abuse pre and postnursing intervention program $(\mathrm{n}=350)$.

\begin{tabular}{|c|c|c|c|c|c|c|}
\hline \multirow[t]{3}{*}{$\mathbf{P}$} & \multirow[t]{3}{*}{$\chi^{2}$} & \multicolumn{4}{|c|}{$\begin{array}{l}\text { The studied secondary school students } \\
(\mathbf{n}=\mathbf{3 5 0})\end{array}$} & \multirow[t]{3}{*}{ Knowledge items about substance abuse } \\
\hline & & \multicolumn{2}{|c|}{ Post-intervention } & \multicolumn{2}{|c|}{ Pre-intervention } & \\
\hline & & $\%$ & $\mathbf{n}$ & $\%$ & n & \\
\hline & & & & & & Definition: \\
\hline \multirow[t]{4}{*}{$0.0001 *$} & 520.94 & 0 & 0 & 54.9 & 192 & Don't know \\
\hline & & 12.3 & 43 & 41.7 & 146 & Incomplete answer \\
\hline & & 87.7 & 307 & 3.4 & 12 & Complete answer \\
\hline & & & & & & Reasons make the individual accustomed it: \\
\hline \multirow[t]{4}{*}{$0.0001 *$} & 471.02 & 0 & 0 & 21.7 & 76 & Don't know \\
\hline & & 14.0 & 49 & 73.4 & 257 & Incomplete answer \\
\hline & & 86.0 & 301 & 4.9 & 17 & Complete answer \\
\hline & & & & & & Symptoms: \\
\hline \multirow[t]{4}{*}{$0.0001 *$} & 662.58 & 0 & 0 & 66.3 & 232 & Don't know \\
\hline & & 2.6 & 9 & 33.4 & 117 & Incomplete answer \\
\hline & & 97.4 & 341 & 0.3 & 1 & Complete answer \\
\hline & & & & & & Resulting damage and risk: \\
\hline \multirow[t]{2}{*}{$0.0001 *$} & 638.80 & 0 & 0 & 56.3 & 197 & Don't know \\
\hline & & 4.9 & 17 & 43.7 & 153 & Incomplete answer \\
\hline
\end{tabular}


Effect of Nursing Intervention for Secondary School Students about Substance Abuse Prevention....

\begin{tabular}{|l|c|c|c|c|c|c|}
\hline & & 95.1 & 333 & 0 & 0 & Complete answer \\
\hline & & & & & & List drinks that contain alcohol: \\
\hline $0.0001^{*}$ & 401.42 & 17.4 & 61 & 48.9 & 171 & Don't know \\
\hline & & 4.6 & 16 & 46.6 & 163 & Incomplete answer \\
\hline & & 78.0 & 273 & 4.6 & 16 & Complete answer \\
\hline & & & & & & Preventive and protective measures against abuse: \\
\hline $0.0001^{*}$ & 551.27 & 0 & 0 & 52.9 & 185 & Don't know \\
\hline & & 13.7 & 48 & 47.1 & 165 & Incomplete answer \\
\hline & & 86.3 & 302 & 0 & 0 & Complete answer \\
\hline
\end{tabular}

Highly statistically significant improvement was found among secondary school students knowledge after the nursing intervention implementation $(\mathrm{P}=0.0001)$.

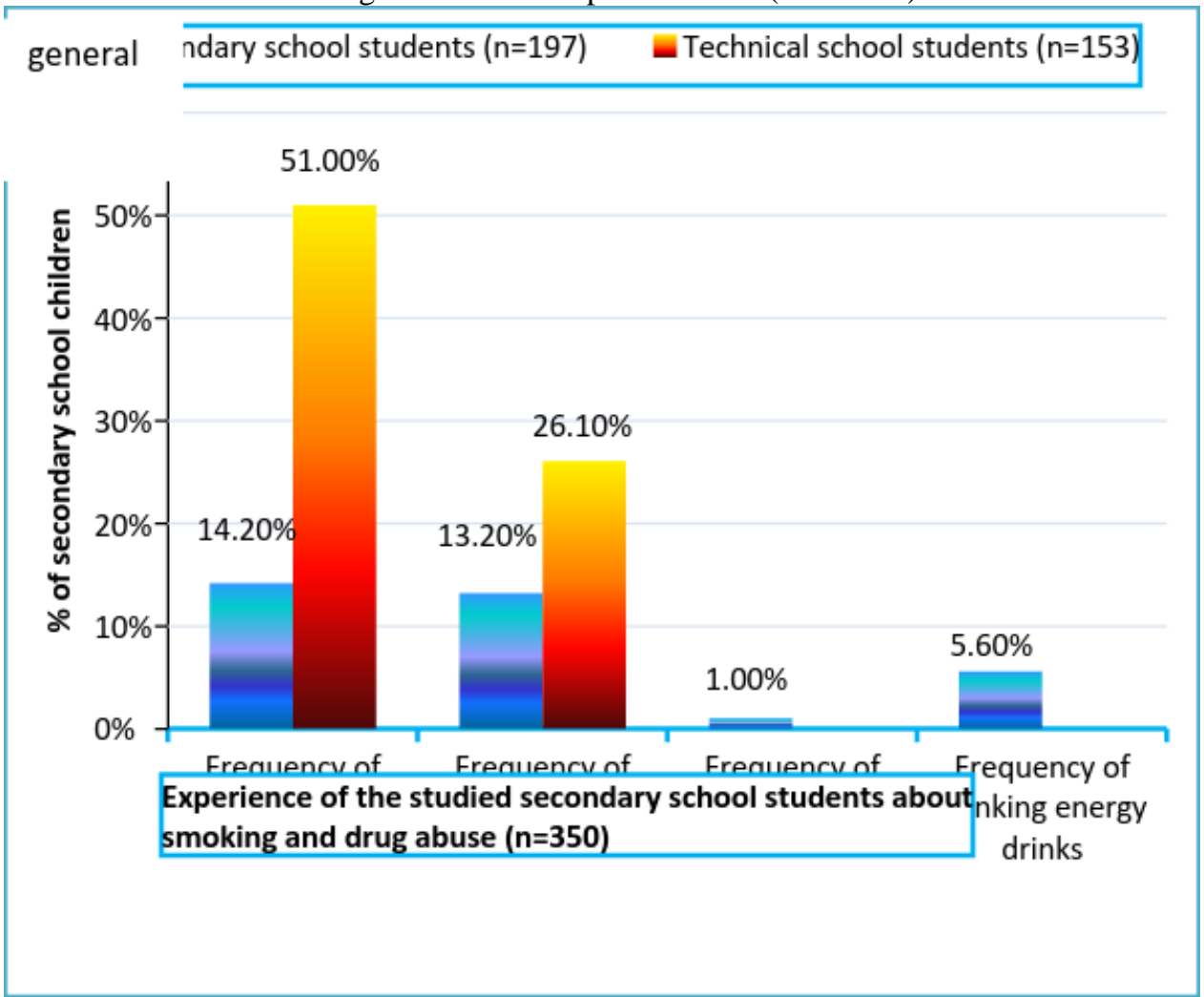

Figure (2): Frequency of the studied secondary school students (general secondary \& technical secondary schools) about smoking and drug abuse $(n=350)$.

Frequency of smoking and drug abuse among the studied secondary school students (general secondary and technical schools), represented $51.0 \%$ and $14.2 \%$ from technical secondary and general secondary school children who smoked cigarette respectively, And for drinking alcohol they accounted for $26.1 \%$ and $13.2 \%$ from technical and general secondary students respectively.

Table (4): Prevalence of abuse among the studied secondary school students (general secondary \& technical secondary schools) $(\mathrm{n}=350)$.

\begin{tabular}{|c|c|c|c|c|c|c|c|c|}
\hline \multirow[t]{3}{*}{$\mathbf{P}$} & \multirow[t]{3}{*}{$\chi^{2}$} & \multicolumn{6}{|c|}{$\begin{array}{l}\text { Frequency of abuse among the studied secondary } \\
\text { school students } \\
(\mathrm{n}=\mathbf{3 5 0})\end{array}$} & \multirow[t]{2}{*}{ Variables } \\
\hline & & \multicolumn{2}{|c|}{$\begin{array}{l}\text { Total } \\
(n=350)\end{array}$} & \multicolumn{2}{|c|}{$\begin{array}{l}\text { Technical } \\
\text { secondary } \\
\text { school } \\
(\mathbf{n}=\mathbf{1 5 3})\end{array}$} & \multicolumn{2}{|c|}{$\begin{array}{l}\text { General } \\
\text { secondary } \\
\text { school } \\
(\mathbf{n}=197) \\
\end{array}$} & \\
\hline & & $\%$ & No & $\%$ & No & $\%$ & No & \\
\hline & & & & & & & & Substance abuse: \\
\hline \multirow[t]{3}{*}{$0.0001^{*}$} & 24.011 & 65.4 & 229 & 51.6 & 79 & 76.1 & 150 & No \\
\hline & & 34.6 & 121 & 48.4 & 74 & 23.9 & 47 & Yes \\
\hline & & & & & & & & -If yes, multiplicity: \\
\hline \multirow[t]{2}{*}{0.217} & 1.521 & 45.9 & 61 & 45.9 & 34 & 57.4 & 27 & Single \\
\hline & & 54.1 & 60 & 40 & 40 & 42.6 & 20 & Multiple \\
\hline
\end{tabular}


Effect of Nursing Intervention for Secondary School Students about Substance Abuse Prevention....

Table (4) demonstrates the prevalence of abuser among the studied secondary school students, where $48.4 \%$ and $23.9 \%$ abuse some drugs in technical secondary and general secondary schools respectively with total prevalence $34.6 \%$.

Table (5): Mean scores and levels of total attitude of the studied secondary school students towards substance abuse pre / post intervention $(n=350)$.

\begin{tabular}{|l|l|l|l|l|l|l|}
\hline \multicolumn{7}{|l|}{$\begin{array}{l}\text { The Studied Secondary School Students } \\
\text { (n=350) }\end{array}$} \\
\hline
\end{tabular}

*Significant $(\mathbf{P}<\mathbf{0 . 0 5})$

Highly statistically significant improvements were found among secondary school students attitude level towards substances abuse and abusers after the intervention $(\mathrm{P}=0.0001)$.

Table (6): Attitude items of the studied secondary school students towards substance abuse ( $\mathrm{n}=350)$.

\begin{tabular}{|c|c|c|c|c|c|c|c|c|}
\hline \multirow[t]{3}{*}{$\begin{array}{ll}\mathbf{2} \\
\mathbf{P}\end{array}$} & \multicolumn{6}{|c|}{$\begin{array}{l}\text { Agreement of the studied secondary school students } \\
(\mathrm{n}=\mathbf{3 5 0})\end{array}$} & & \multirow[t]{3}{*}{ Attitude items towards substance abuse } \\
\hline & \multicolumn{2}{|c|}{ Agree } & \multicolumn{2}{|c|}{ Neutral } & \multicolumn{2}{|c|}{ Disagree } & & \\
\hline & $\%$ & $\mathrm{n}$ & $\%$ & $\mathrm{n}$ & $\%$ & $\mathrm{n}$ & & \\
\hline 132.38 & 67.7 & 237 & 0 & 0 & 32.3 & 113 & Pre & It is harmful to health \\
\hline $0.0001 *$ & 100 & 350 & 0 & 0 & 0 & 0 & Post & \\
\hline 627.91 & 90.6 & 317 & 4.0 & 14 & 5.4 & 19 & Pre & It activates the body \\
\hline $0.0001 *$ & 0 & 0 & 0 & 0 & 100 & 350 & Post & \\
\hline 200.92 & 32.9 & 115 & 0 & 0 & 67.1 & 235 & Pre & It is a manifestation of manhood \\
\hline $0.0001 *$ & 0 & 0 & 24.0 & 84 & 76.0 & 266 & Post & \\
\hline 256.79 & 43.1 & 151 & 0 & 0 & 56.9 & 199 & Pre & $\begin{array}{l}\text { It is a boredom for human or away boring for } \\
\text { human }\end{array}$ \\
\hline $0.0001^{*}$ & 0 & 0 & 28.6 & 100 & 71.4 & 250 & Post & \\
\hline 184.348 & 38.6 & 135 & 3.1 & 11 & 58.3 & 204 & Pre & Believe that it increases the ability to \\
\hline $0.0001^{*}$ & 0 & 0 & 0 & 0 & 100 & 350 & Post & studying \\
\hline 684.18 & 1.1 & 4 & 32.3 & 113 & 66.6 & 233 & Pre & Its use in times of testing is not harmful \\
\hline $0.0001 *$ & 100 & 350 & 0 & 0 & 0 & 0 & Post & \\
\hline 81.49 & 1.1 & 4 & 37.7 & 132 & 61.1 & 214 & Pre & $\begin{array}{l}\text { Its use helps to high rate of achievement in } \\
\text { studying }\end{array}$ \\
\hline $0.0001 *$ & 0 & 0 & 9.7 & 34 & 90.3 & 316 & Post & \\
\hline 141.92 & 66.3 & 232 & 32.3 & 113 & 1.4 & 5 & Pre & Its use leads to bad psychological effects \\
\hline $0.0001 *$ & 100 & 350 & 0 & 0 & 0 & 0 & Post & \\
\hline
\end{tabular}


Effect of Nursing Intervention for Secondary School Students about Substance Abuse Prevention....

\begin{tabular}{|c|c|c|c|c|c|c|c|l|}
\hline 457.91 & 1.1 & 4 & 79.1 & 277 & 19.7 & 69 & Pre & $\begin{array}{l}\text { The use of drugs, cigarettes does not lead to } \\
\text { addiction }\end{array}$ \\
\hline $0.0001^{*}$ & 0 & 0 & 0.9 & 3 & 99.1 & 347 & Post & Pre \\
\hline 216.51 & 9.1 & 32 & 41.4 & 145 & 49.4 & 173 & $\begin{array}{l}\text { Its use is incompatible with the values and } \\
\text { standards of the society }\end{array}$ \\
\hline $0.0001^{*}$ & 0 & 0 & 1.7 & 6 & 98.3 & 344 & Post & \\
\hline 167.48 & 38.3 & 134 & 0 & 0 & 61.7 & 216 & Pre & $\begin{array}{l}\text { Substance abuse or stimulants serves to activate } \\
\text { students mind }\end{array}$ \\
\hline $0.0001^{*}$ & 0 & 0 & 0.9 & 3 & 99.1 & 347 & Post & \\
\hline 46.80 & 1.7 & 6 & 32.3 & 113 & 66.0 & 231 & Pre & It is supposed to allow the use of narcotic pills \\
\hline $0.0001^{*}$ & 0 & 0 & 12.6 & 44 & 87.4 & 306 & Post & \\
\hline 139.14 & 63.4 & 222 & 3.7 & 13 & 32.9 & 115 & Pre & $\begin{array}{l}\text { Strong punishment must be applied for drug } \\
\text { abusers }\end{array}$ \\
\hline $0.0001^{*}$ & 91.7 & 321 & 8.3 & 29 & 0 & 0 & Post & \\
\hline
\end{tabular}

Highly statistically significant improvements were found among secondary school students attitude level towards substances abuse after the intervention $(\mathrm{P}=0.0001)$.

Table (7): Attitude of the studied secondary school students towards substance or drug abuser $(\mathrm{n}=350)$.

\begin{tabular}{|c|c|c|c|c|c|c|c|c|}
\hline \multirow[t]{3}{*}{$\begin{array}{l}\chi^{2} \\
\mathbf{P} \\
\end{array}$} & \multicolumn{6}{|c|}{$\begin{array}{c}\text { Agreement of the studied secondary school students } \\
(n=350)\end{array}$} & & \multirow[t]{3}{*}{ Attitude items towards drug abuser } \\
\hline & \multicolumn{2}{|c|}{ Agree } & \multicolumn{2}{|c|}{ Neutral } & \multicolumn{2}{|c|}{ Disagree } & & \\
\hline & $\%$ & $\mathbf{n}$ & $\%$ & $\mathbf{n}$ & $\%$ & $\mathbf{n}$ & & \\
\hline 329.41 & 63.4 & 222 & 0.6 & 2 & 36.0 & 126 & Pre & $\mathrm{He}$ is a failed person \\
\hline $0.0001 *$ & 0 & 0 & 0 & 0 & 100 & 350 & Post & \\
\hline 171.89 & 60.6 & 212 & 33.4 & 117 & 6.0 & 21 & Pre & I don't allow myself to mingle with members \\
\hline $0.0001 *$ & 100 & 350 & 0 & 0 & 0 & 0 & Post & of using narcotic pills \\
\hline 187.19 & 58.6 & 205 & 1.1 & 4 & 40.3 & 141 & Pre & Strongly refuse to marry a girl who has \\
\hline $0.0001^{*}$ & 90.9 & 318 & 9.1 & 32 & 0 & 0 & Post & addicted brother \\
\hline 84.73 & 63.4 & 222 & 29.1 & 102 & 7.4 & 26 & Pre & Strongly refuse to marry one of relative of a \\
\hline $0.0001 *$ & 91.7 & 321 & 8.3 & 29 & 0 & 0 & Post & person who uses drugs \\
\hline 156.73 & 57.7 & 202 & 5.7 & 20 & 36.6 & 128 & Pre & If the addict is a relative I will feel shame \\
\hline $0.0001 *$ & 91.7 & 321 & 8.3 & 29 & 0 & 0 & Post & \\
\hline 77.61 & 64.9 & 227 & 29.4 & 103 & 5.7 & 20 & Pre & I cannot trust in a person who uses narcotic \\
\hline $0.0001^{*}$ & 91.7 & 321 & 8.3 & 29 & 0 & 0 & Post & pills \\
\hline 22.23 & 94.0 & 329 & 0.3 & 1 & 5.7 & 20 & Pre & People who abuse drugs must be treated \\
\hline $0.0001^{*}$ & 98.9 & 346 & 1.1 & 4 & 0 & 0 & Post & instead of punishment \\
\hline 153.19 & 64.0 & 224 & 0.6 & 2 & 35.4 & 124 & Pre & Person who uses narcotic drugs find it \\
\hline $0.0001 *$ & 97.1 & 340 & 2.9 & 10 & 0 & 0 & Post & difficult to live in a normal social life \\
\hline 161.49 & 63.7 & 223 & 1.4 & 5 & 34.9 & 122 & Pre & Person who uses narcotic drugs should be \\
\hline $0.0001 *$ & 89.4 & 313 & 10.6 & 37 & 0 & 0 & Post & ashamed of himself \\
\hline 299.27 & 32.0 & 112 & 54.0 & 189 & 14.0 & 49 & Pre & If I have a friend abuse drugs, the \\
\hline $0.0001^{*}$ & 94.9 & 332 & 5.1 & 18 & 0 & 0 & Post & relationship with him will continue \\
\hline 62.70 & 65.4 & 229 & 28.9 & 101 & 5.7 & 20 & Pre & I am ashamed to appear in public places, \\
\hline $0.0001 *$ & 89.4 & 313 & 10.6 & 37 & 0 & 0 & Post & accompanied by someone who is known as drug abuser \\
\hline 63.75 & 65.1 & 228 & 29.1 & 102 & 5.7 & 20 & Pre & I will be strongly bothered if my brother uses \\
\hline $0.0001 *$ & 89.4 & 313 & 10.6 & 37 & 0 & 0 & Post & drugs \\
\hline 66.23 & 64.3 & 225 & 30.0 & 105 & 5.7 & 20 & Pre & Do not allow my family members to be \\
\hline $0.0001 *$ & 89.4 & 313 & 10.6 & 37 & 0 & 0 & Post & accompanied by addicted man \\
\hline 482.26 & 15.1 & 53 & 50.6 & 177 & 34.3 & 120 & Pre & Addicted person is apathetic \\
\hline $0.0001 *$ & 97.4 & 341 & 2.6 & 9 & 0 & 0 & Post & \\
\hline 153.55 & 60.5 & 212 & 4.9 & 17 & 34.6 & 121 & Pre & Addicted person does not have ambition \\
\hline $0.0001 *$ & 97.4 & 341 & 2.6 & 9 & 0 & 0 & Post & \\
\hline 124.69 & 64.3 & 225 & 29.1 & 102 & 6.6 & 23 & Pre & Addicted person cannot succeed in any \\
\hline $0.0001 *$ & 97.4 & 341 & 2.6 & 9 & 0 & 0 & Post & business \\
\hline 631.52 & 5.1 & 18 & 82.3 & 288 & 12.6 & 44 & Pre & A person who uses drugs can take off them \\
\hline $0.0001^{*}$ & 100 & 350 & 0 & 0 & 0 & 0 & Post & without need for treatment \\
\hline 50.38 & 86.6 & 303 & 7.7 & 27 & 5.7 & 20 & Pre & Normal person doesn't use narcotic drugs \\
\hline $0.0001 *$ & 100 & 350 & 0 & 0 & 0 & 0 & Post & \\
\hline
\end{tabular}

Highly statistically significant improvements were found among secondary school students attitude level towards abusers after the intervention $(\mathrm{P}=0.0001)$.

Table (8): Mean scores of total knowledge and attitude towards substance abuse of the studied secondary school students in relation to their demographic data pre-intervention $(\mathrm{n}=350)$.

\begin{tabular}{|l|l|l|l|ll|}
\hline \multicolumn{2}{|c|}{ The Studied Secondary School Students Pre-intervention $(\mathbf{n}=350)$} & Variables \\
\hline $\begin{array}{c}\text { t-test or } \\
\text { F value } \\
\text { P- value }\end{array}$ & $\begin{array}{l}\text { Total attitude } \\
\text { scores } \\
\text { Mean } \pm \text { SD }\end{array}$ & $\begin{array}{l}\text { t-test or } \\
\text { F value } \\
\text { P- value }\end{array}$ & $\begin{array}{l}\text { Total knowledge } \\
\text { scores } \\
\text { Mean } \pm \text { SD }\end{array}$ & \\
\hline & & & & School type: & \\
\hline 12.063 & $40.64 \pm 4.47$ & 11.822 & $5.51 \pm 1.43$ & General secondary \\
\hline
\end{tabular}


Effect of Nursing Intervention for Secondary School Students about Substance Abuse Prevention....

\begin{tabular}{|c|c|c|c|c|}
\hline & & & & school \\
\hline \multirow[t]{2}{*}{$0.0001 *$} & $32.01 \pm 8.67$ & $0.0001 *$ & $3.15 \pm 2.29$ & $\begin{array}{l}\text { Technical secondary } \\
\text { school }\end{array}$ \\
\hline & & & & Fathers' education level: \\
\hline 23.103 & $32.71 \pm 7.18$ & 22.953 & $2.00 \pm 2.00$ & Illiterate \\
\hline \multirow[t]{5}{*}{$0.0001 *$} & $30.28 \pm 1.92$ & $0.0001 *$ & $2.17 \pm 1.93$ & Read \& write \\
\hline & $30.00 \pm 0.00$ & & $4.27 \pm 2.31$ & Basic education \\
\hline & $39.35 \pm 6.82$ & & $4.89 \pm 1.63$ & Middle education \\
\hline & $38.36 \pm 8.86$ & & $5.02 \pm 2.17$ & University education \\
\hline & & & & Mothers' education level: \\
\hline 33.027 & $30.00 \pm 0.00$ & 19.136 & 0.00 & Illiterate \\
\hline \multirow[t]{5}{*}{$0.0001 *$} & $30.00 \pm 0.00$ & $0.0001 *$ & $3.22 \pm 1.70$ & Read \& write \\
\hline & $30.82 \pm 3.38$ & & $3.44 \pm 2.54$ & Basic education \\
\hline & $35.90 \pm 9.60$ & & $4.16 \pm 2.33$ & Middle education \\
\hline & $41.93 \pm 1.71$ & & $5.64 \pm 1.26$ & University education \\
\hline & & & & Residence: \\
\hline 1.744 & $37.77 \pm 6.20$ & 1.437 & $4.69 \pm 1.77$ & Rural \\
\hline 0.082 & $36.27 \pm 8.79$ & 0.152 & $4.34 \pm 2.42$ & Urban \\
\hline
\end{tabular}

Statistically, significant positive relationships were detected between score of total knowledge and attitude scores of secondary school students with some of demographic characteristics as school type, mothers and fathers educational level.

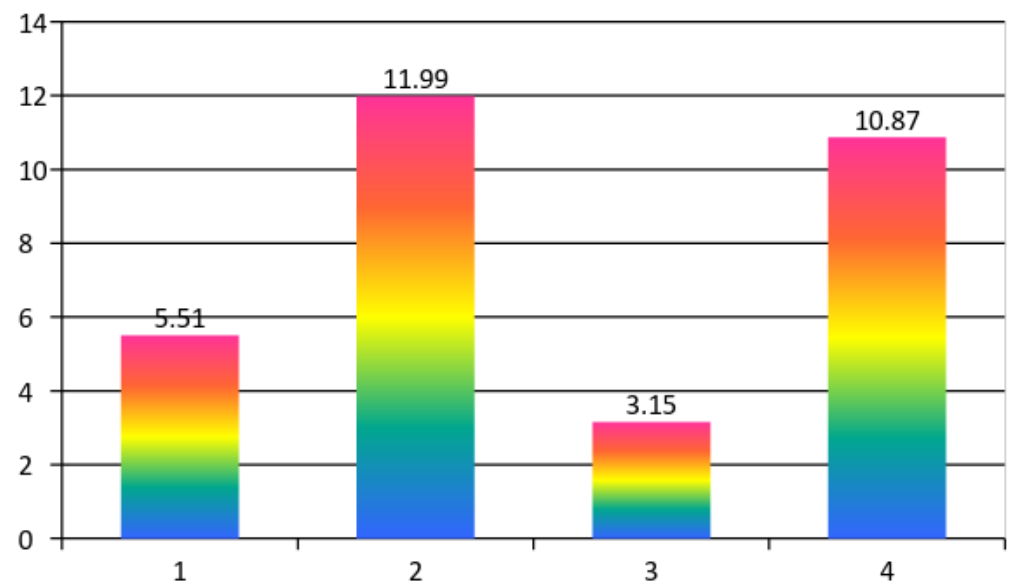

Figure (3): Change in scores of total knowledge about substance abuse of the studied secondary school students (general secondary \& technical secondary schools) in relation to their type of school pre/ post Intervention $(n=350)$.

Figure (3) shows significant change of total knowledge scores about substance abuse of the studied secondary school students (general secondary \& technical schools) in relation to their type of school pre/post intervention.

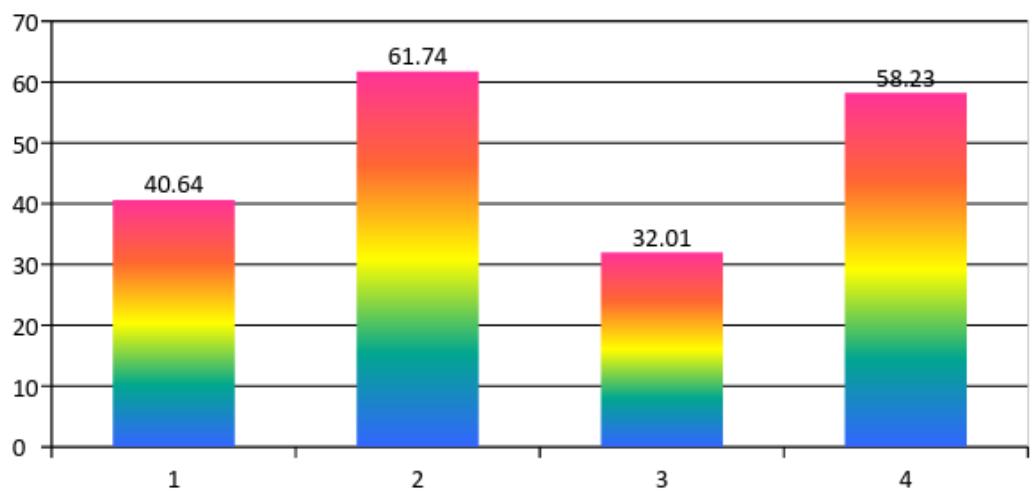

Figure (4): Change in scores of total attitude towards substance abuse and abusers of the studied secondary school students (general secondary \& technical secondary schools) in relation to their type of school pre/ post intervention $(\mathrm{n}=350)$. 
Figure (4) shows significant positive changes of total attitude scores about substance abuse and abusers of the studied secondary school students (general secondary \& technical secondary schools) post intervention

Table (9): Correlation between levels of total knowledge and total attitude about substance abuse among the studied secondary school students pre / post- intervention implementation $(n=350)$.

\begin{tabular}{|c|c|c|c|c|c|c|c|c|c|}
\hline \multirow{4}{*}{$\begin{array}{l}\chi^{2} \\
\mathbf{P}\end{array}$} & \multicolumn{8}{|c|}{$\begin{array}{l}\text { Total Knowledge Levels of the Studied Secondary School Students } \\
(n=350)\end{array}$} & \multirow{4}{*}{$\begin{array}{lrr}\text { Levels } & \text { of } & \text { Total } \\
\text { Attitude } & \text { Towards } \\
\text { Substance } & \text { Abuse and } \\
\text { Abusers } & \\
\end{array}$} \\
\hline & \multicolumn{4}{|c|}{ Post-program } & \multicolumn{4}{|c|}{ Pre-program } & \\
\hline & \multicolumn{2}{|c|}{$\begin{array}{l}\text { Good } \\
\text { (302) }\end{array}$} & \multicolumn{2}{|c|}{$\begin{array}{l}\text { Fair } \\
(n=48)\end{array}$} & \multicolumn{2}{|c|}{$\begin{array}{l}\text { Fair } \\
(n=47)\end{array}$} & \multicolumn{2}{|c|}{$\begin{array}{l}\text { Poor } \\
(n=303)\end{array}$} & \\
\hline & & & $\%$ & No & & & $\%$ & No & \\
\hline & & & & & & & & & Preprogram: \\
\hline 18.929 & & & & & 0 & 0 & 6.3 & 19 & Negative \\
\hline \multirow[t]{4}{*}{$0.0001^{*}$} & & & & & 8.5 & 4 & 35.0 & 106 & Neutral \\
\hline & & & & & 91.5 & 43 & 58.7 & 178 & Positive \\
\hline & & & & & \multicolumn{4}{|c|}{$0.6760 .0001 *$} & R P \\
\hline & & & & & & & & & Post program: \\
\hline \multirow[t]{2}{*}{-} & 100 & 302 & 100 & 48 & & & & & Positive \\
\hline & \multicolumn{4}{|c|}{$0.6870 .0001 *$} & & & & & R P \\
\hline
\end{tabular}

*Significant $(\mathbf{P}<\mathbf{0 . 0 5})$

$r=$ Correlation Coefficient

Statistically significant positive correlations were detected between changes of attitude scores of secondary school students with changes in knowledge scores post intervention $(\mathrm{P}=0.001)$.

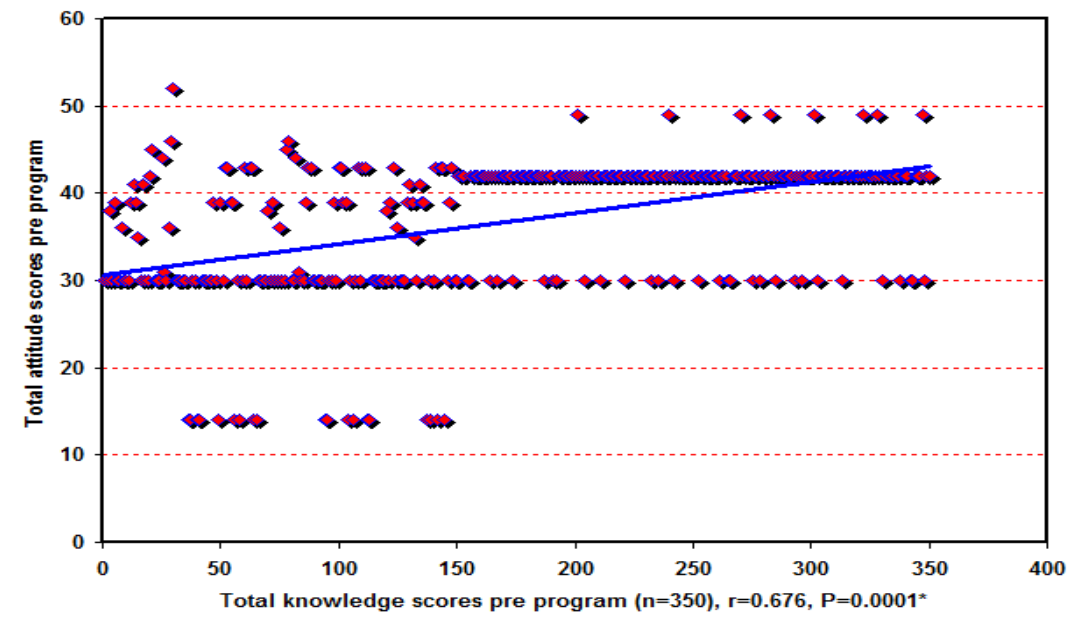

Figure (5): Correlations between total knowledge scores and total attitude scores about substance abuse among the studied secondary school students pre nursing intervention $(n=350)$.

Figure (5) illustrate statistically, significant positive correlations were detected between attitude scores of secondary school students with knowledge scores (0.001) pre/intervention.

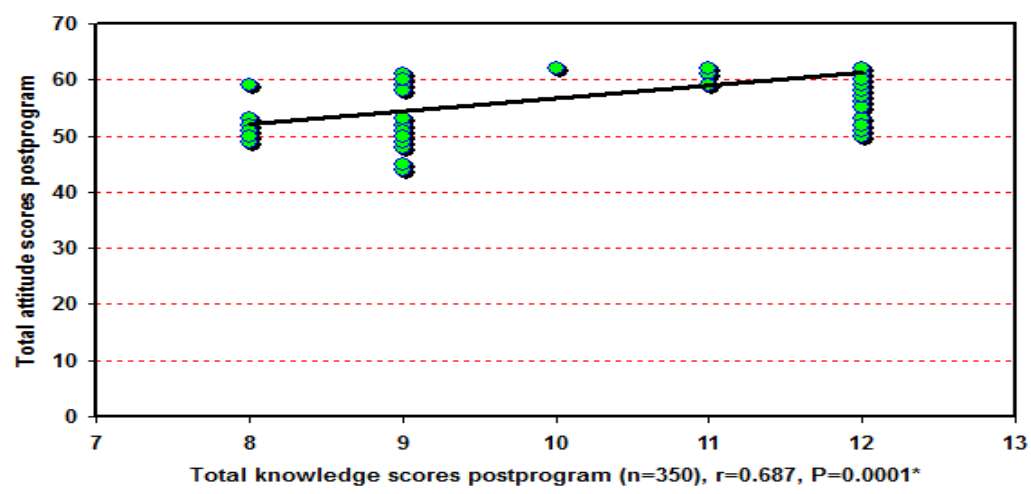

Figure (6): Correlations between total knowledge scores and total attitude scores about substance abuse among the studied secondary school students post/ intervention $(n=350)$. 
Figure (6) represent statistically, significant positive correlations were detected between changes of attitude scores of secondary school students with changes in knowledge scores post intervention $(\mathrm{P}=0.001)$.

\section{Discussion}

Although most young people who experiment alcohol and other drugs don't encounter major issues, drug use can cause many and varied problems. Using legal or illegal drugs may influence the young people themselves, as well as friends, family and others around them. The short term risks of alcohol and other drug use incorporate risk of injury, loss of belonging, relationship issues, failed in school or work, and maybe even trouble with the law. The more extended term risks, on the other hand, include the risk of developmental problems, reliance, and chronic health problems (Gabrhelik et al., 2011) ${ }^{\mathbf{1 0}}$.

Comparing knowledge levels of secondary school students ( general secondary vs. technical secondary schools), regarding substance abuse, finding revealed that pre-intervention the mean knowledge score for technical school students was 3.15 \pm 2.29 , while for general secondary school students, it was higher (5.51 \pm 1.43 ). From the researcher point of view that is may be due to that general school students are characterized by that they are more committed, familiar with information and more anxious to acquire information.

Pre- intervention, majority of the total sample had poor level of total knowledge score, regarding substance abuse, while more than tenth had fair level and none of them had good knowledge. These study findings are in accordance with Haddad et al. (2010) ${ }^{\mathbf{1 1}}$ Jordan, who found some gaps in students' knowledge, attitudes and beliefs. As well, Nebhinani et al. (2013) ${ }^{\mathbf{1 2}}$ India, found that greater number of students had sufficient knowledge about harmful effects of addictive substances but had constrained data regarding treatment alternatives.

The current study results were less similar to those reported by Geramian et al. (2012) ${ }^{\mathbf{1 3}}$ Isfahan Province Health Center, Iran, who asserted that knowledge level of high school students about addictive drugs and particularly their adverse impacts is in a medium level for both genders and in urban and provincial zone.

Considering the total attitude level of the studied school students, toward substance abuse, less than three fifth of them had negative attitude, while more than two fifth had neutral attitude, and a minority had a positive attitude. However students' attitude toward substance abuser results revealed that almost two third had positive attitude, while more than one third had negative attitude and none of them had neutral attitude. These finding are supported by Al-Dossary (2004) ${ }^{\mathbf{9}}$ Wadi Al-Dawasser, Saudi Arabia, who reported that most the attitudes of the research individuals inclined to the negative attitude towards the stimulate drugs. In a similar study Prakash et al. (2009) ${ }^{\mathbf{1 4}}$ India, expressed that their attitude towards addiction seemed to have many blemishes and most of the students appeared to have sufficient information about addictive substance however not in depth.

Regarding the change of total knowledge and total attitude about substance abuse among the studied secondary school students pre/post-intervention statistically significant positive correlations were detected between change of attitude scores of secondary school students with change in knowledge scores $(0.001)$. This finding is supported by Giannetti et al. (2008) ${ }^{\mathbf{1 5}}$ Duquesne University who guaranteed that several studies in youth population have reported positive relationship between knowledge about substance abuse and their attitudes toward substances.

Concerning frequency of smoking and drug abuse among the studied school students (technical and general secondary), cigarette smoking showed the highest prevalence among both schools with higher rate among technical school students, followed by alcohol intake with higher rate among technical school students. However, the least was energy drinks and inhaling products among both schools, with total rate prevalence representing more than one third. From the researcher point of view that is may be related to the cheap price of cigarettes from the other type of the substance abuse next to it is the most widespread and commonly used between the Egyptian population that may reflect the influence of cultural and economic factors. These study findings are to some extent in accordance with those of a study carried out by Ranjan et al.( 2010) ${ }^{\mathbf{1 6}}$, in Mumbai who found that smokeless tobacco accounted for $43.7 \%$ followed by smoking tobacco (21\%), then Alcohol $(12.2 \%)$. As well the Department for National Drug Control (2013) ${ }^{17}$, Bermuda, and Kotina et al. $(2016)^{18}$ Malla Reddy hospital India reported that alcohol intake was high showing prevalence of $48.54 \%$, followed by cigarette smoking $(23.36 \%)$, then tobacco $(8.76 \%)$, pan $(3.65 \%)$, toddy $(6.93 \%)$, guthka $(6.93 \%)$, and khaini $(2.2 \%)$.

However Johnston et al. (2011) ${ }^{\mathbf{1 9}}$, University of Michigan reported that overall, $76 \%(2,418)$ of all survey respondents have reported use of at least one drug in their lifetime. Students recorded the highest lifetime prevalence-of-use for energy drinks (65.5\%), alcohol (54.9\%), marijuana (21.2\%), inhalants $(12.1 \%)$, and cigarettes $(10.7 \%)$.

Concerning students' sources of information about substance abuse, the highest percentage of students in the present study, these were friends followed by Net, TV, parents, and minorities as experiences, schools, 
mosques and churches. Similar results were mentioned by several studies as; Tsering et al. (2010) ${ }^{\mathbf{2 0}}$ India, and Salim and Siddiqui (2015) ${ }^{21}$ Jizan city, Kingdom of Saudi Arabia.

Regarding the effect of nursing intervention about substance abuse on student findings revealed that highly statistically significant improvements on school students knowledge and attitude after the intervention implementation $(\mathrm{P}=0.0001)$. This improvement may be due to knowledge refreshment through the intervention sessions and relevance of items of the intervention content. As reported by Newton et al.( 2009a,b) ${ }^{\mathbf{2 2 , 2 3}}$ Australia evaluation of the findings state that in comparison with usual drug education programs, students in the intervention group showed significantly greater enhancements in alcohol and cannabis knowledge at the end of the course, additionally at six and twelve months taking after the intervention. As well, Spoth et al.( 2009) ${ }^{\mathbf{2 4}}$ Iowa State University and Faggianoa et al.( 2010) ${ }^{25}$ in Seven European countries provided support for the efficacy of school-based programs, at least in the short term (defined as at least 6 months after the intervention was implemented).

Kerry et al. (2013) ${ }^{\mathbf{2 6}}$, UK reported substantial evidence relating to the positive effects of school-based alcohol education and life-skills programs on school children's alcohol related-knowledge and attitude.

\section{Conclusion}

The nursing intervention helped in improving students' knowledge, awareness, and their attitude toward substance abuse, and abuser.

\section{Recommendations}

There is a need for continued and repeated more education for students at an earlier age than traditionally provided. Parents should also receive information on substances used by young people, the associated dangers with use, and the parents' role in prevention of problem substance use. Further study to be conducted in other settings and more schools to know the prevalence of drug abuse, measures used to prevent its occurrence and generalization.

\section{References}

[1]. UNICEF report (2011): Demographic trends for adolescents' ten key facts. [Cited 2011 November 10]; Available from: http://www.unicef.org/ sowc2011/pdfs/Demographic-Trends.pdf.

[2]. Chatterjee,S., Tempalski,B., Pouget, E.R., Cooper,H.L., Cleland,C.M., \& Friedman, S.R. (2011): Changes in the prevalence of injection drug use among adolescents and young adults in large U.S. Metropolitan Areas. AIDS and Behavior; 15:15 70-1578.

[3]. James, C. (2013): Drug prevention programmes in schools: What is the evidence? Montour UK; Available at www.mentoruk.org.uk.

[4]. World Health Statistics. (2010): Available from: www.who. Int/whosis/who stat/EN_WHS08_Full.pdf.

[5]. Ferrante,M., Saulle, R., Ledda, C., Pappalard, R., Fallico, R., Torre, G., \& Fiore, M. (2013): Prevalence of smoking habits, attitudes, knowledge and beliefs among health professional school students: a cross-sectional study. Ann Ist Super Sanità; 49(2):143-149.

[6]. Matt, V. (2014): 8 Substance dependence and abuse Nursing care plans Mar 10, 2014 Enjoys health technology and innovations about nursing and medicine in general; Available at https://nurseslabs.com/.

[7]. Alibrahim,O., Elawad, N., Misau, Y.A., Shaikh,T.M., Allam, N.(2012): Psychotic symptoms: a retrospective study of adolescents who abuse drugs at Al-Amal Hospital in Jeddah, Saudi Arabia. Journal of Public health in Africa;3:e5.

[8]. Dawson,B.D., \& Trapp,R.G. (2001): Reading the medical literature: Basic \& Clinical Biostatistics. Lange Medical Book/ McGraw-Hill. Medical Publication Division, New York. ( $3^{\text {rd }}$ ed) Ch. 7-9, Pp. 161-218 and Ch. 13, 305-314.

[9]. Al-Dossary, S.M.T. (2004): The attitudes of the teachers and the students of secondary schools in the Wadi Al-Dawasser towards the stimulated drugs. Naif Arab University for Security Science

[10]. Gabrhelik, R., Duncan, A., Miovsky, M.C, Debra, M., Furr,H., Stastna, L., \&, L. (2011): "Unplugged”: A school-based randomized control trial to prevent and reduce adolescent substance use in the Czech Republic; Accepted: December, 14, 2011;Received in revised form: December, 12, 2011; Received: May, 24, 2011

[11]. Haddad, L., Shotar, A., Umlauf, M., \& Al-Zyoud, S. (2010): Knowledge of substance abuse among high school students in Jordan. Journal of Transcultural Nursing; 21:143-150.

[12]. Nebhinani, N., Nebhinani, M., Kumar, A.M., \& Grewal, S. (2013): Substance-related knowledge and attitude in school and college students. German J Psychiatry; 16(1): 15-19.

[13]. Geramian, N., Akhavan, S., \& Gharaat, L. (2012): Determinants of drug abuse in high school students and their related knowledge and attitude. Journal of Pakistan Medical Association; March 62(3) Suppl. 1.

[14]. Prakash, O., Giri, O.P., Mishra, A.K., Kumar, S., \& Kulhara, P. (2009): Knowledge and attitude of Indian adolescents towards addiction: Finding from an exploratory survey. J Mental Health \& Human Behavior; 14:74-79.

[15]. Giannetti, V.J., Sieppert, J.D., \&Holosko, M.J. (2008): Attitudes and knowledge concerning alcohol abuse: Curriculum implications. Journal of Health and Social Policy Published online: 21 Oct 2008; 15:45-58.

[16]. Ranjan, D.P., Namita, R., \& Chaturvedi, M. (2010): A study of prevalence of drug abuse in aged 15 years \& above in the urban slum community of Mumbai Indian J. Prev. Soc. Med; vol.41 :( 1, 2).

[17]. Department for National Drug Control (2013): Report of the Survey of Students on Knowledge and Attitudes of Drugs and Health, 2012. Government of Bermuda.

[18]. Kotina, S., Gokuldas, V., Sawant, P. R., \& Kokiwar.(2016): A study to determine causes, prevalence and knowledge regarding consequences of substance abuse: A community based cross sectional study International Journal of Community Medicine and Public Health, Mar ;3(3):730-734 from http://www.ijcmph.com 
[19]. Johnston, L. D., O’Malley, P. M., Bachman, J. G., \& Schulenberg, J. E. (2011): Monitoring the future national survey results on drug use, 1975-2010: Volume 1, Secondary School Students. Ann Arbor: Institute for Social Research. The University of Michigan. Retrieved January 28, 2012, from http://monitoringthefuture.org/pubs.html

[20]. Tsering, D., Pal, R., \& Dasgupta, A. (2010): Substance use among adolescent high school students in India: A survey of knowledge, attitude and opinion. J Pharmacy and Bioallied Sciences; 2:137-40.

[21]. Salim, A.M.A., \& Siddiqui, A.F. (2015): Substance Abuse, A Public Health Challenge: Study From Jizan City, Saudi Arabia People's Journal of Scientific Research, July; 8: 2.

[22]. Newton, N.C., Andrews, G., Teesson, M., \& Vogl, L.E. (2009a). Delivering prevention for alcohol and cannabis using the internet: A cluster randomised controlled trial. Preventive Medicine; 48: 579-584.

[23]. Newton, N.C., Teesson, M., Vogl, L., \& Andrews, G. (2009b): Internet-based prevention for alcohol and cannabis use: Final results of the climate schools alcohol and cannabis course. Addiction, Accepted 21 st October 2009.

[24]. Spoth, R., Greenberg, M., \& Turrisi, R. (2009): Overview of preventive intervention addressing underage drinking: State of the evidence and steps toward public health impact. Alcohol Research \& Health; 32:53-66.

[25]. Faggianoa, F.B., Tagliantia, F.V., Burkhartc, G., Bohrnd, K., Cuomob, L., Gregorie, D., Panellaa, M., Scatignaf, M., Siliquini, E.R., Varonag, L., Kreefth, Pv., \& Vassarai, M. (2010): The effectiveness of a school-based substance abuse prevention program: 18-month follow-up of the EU-Dap cluster randomized controlled trial Drug and Alcohol Dependence GModel DAD-3692; No. of Pages 92010 Published by Elsevier Ireland Ltd.

[26]. Kerry, M., Nelson, J. \& Lynch, S. (2013). Effectiveness of school-based life-skills and alcohol education programmes: A review of the literature. Slough: NFER. National Foundation for Educational Research. 